\title{
Etude comparative entre la température expérimentale et simulée d'un couple acier-carbure de tungstène animé d'un mouvement de rotation
}

\author{
Nafissa. Khennafi-Benghalem ${ }^{\mathrm{a}, \mathrm{b}}$, Naserddine Sabri ${ }^{\mathrm{b}}$ \\ Unité de recherche des matériaux émergents ${ }^{\mathrm{a}}$ \\ Institut d'optique et de mécanique de précision ${ }^{b}$ \\ Université Ferhat Abbas Sétif 1 Algérie
}

\begin{abstract}
Abstrait. De toutes les grandeurs physiques, la température est certainement l'une de celles dont la mesure est la plus fréquente, la température détermine en effet de façon décisive les propriétés de la matière, c'est pourquoi, on recherche comme dans l'industrie et les laboratoires, la mesure et le contrôle très strict des températures sont indispensables. Nous avons réalisé deux séries d'essais d'usure d'un couple acier-carbure de tungstène animé d'un mouvement de rotation. La force normale $\mathrm{P}$ est égale à environ 500 et $1000 \mathrm{~N}$ respectivement pour la série 1 et 2 , la température est prise dans l'intervalle $\left[20-200^{\circ} \mathrm{C}\right]$ et la vitesse de glissement fixée à $\mathrm{v}=0.1 \mathrm{~m} / \mathrm{s}$. La température indiquée est celle relevée par un capteur inséré au milieu de l'éprouvette de forme conique et à $2 \mathrm{~mm}$ du contact. Pour chaque température, deux essais ont été effectués sur deux éprouvettes différentes. La longueur de glissement est de $\mathrm{L}=90 \mathrm{~m}$. L'ordre de grandeur de la vitesse d'usure est de quelques $10^{-5} \mathrm{~mm}^{3} . \mathrm{N}^{-1} . \mathrm{m}^{-}$ ${ }^{1}$. Le coefficient de frottement se situe entre 0.12 et 0.8 . L'objectif de ce présent travail est de comparer entre la température expérimentale de ce couple acier-carbure de tungstène en mouvement, mesurée au cours de l'essai et celle simulée en fonction du coefficient de frottement et pour recueillir une information supplémentaire plus exactement sur la température au point de contact.
\end{abstract}

\section{Introduction}

La connaissance de la température dans un contact frottant est nécessaire afin d'étudier son influence sur le comportement en frottement et sur les mécanismes de dégradation des surfaces. [1]. La mesure directe n'est cependant pas aisée; la zone d'intérêt étant sollicitée mécaniquement, l'instrumentation par des thermocouples surfaciques résiste peu aux contraintes générées par le frottement [2]. Cependant la mesure de la température est très difficile à effectuer compte tenu du choix de l'endroit de prise de la température et des phénomènes d'évacuation de la chaleur et de la dissipation de l'énergie [3]. La mesure par des techniques sans contact telles que pyromètres ou caméra radiométrique n'est pas réalisable au sein du contact pour des matériaux opaques [4]. Ces dernières sont également tributaires des variations de l'émissivité au cours de l'essai et l'information est erronée si celle-ci ne peut pas être estimée simultanément à la température. Le développement des moyens informatiques permet aujourd'hui de recourir à des techniques numériques pour identifier l'énergie dissipée de part et d'autre du contact et les températures à l'interface [5]. Le développement des techniques numériques a permis d'implémenter les caractéristiques mécaniques et thermiques des matériaux frottant dans les logiciels de calcul par éléments finis [6]. Ces modèles sont basés sur une loi d'Archard modifiée donnant le taux d'usure en fonction de la distance parcourue sur la surface, de l'effort appliqué, du coefficient de frottement et de paramètres propres aux matériaux étudiés. Le calcul du champ thermique au sein des éléments frottant fait l'objet de nombreux développements analytiques. Siroux et al. [7] ont déterminé la température surfacique du disque à partir de mesures dans la masse et l'ont comparée à une mesure pyrométrique. McColl [8] a simulé le contact cylindre-plan de type fretting pour un couple de matériaux alliage d'acier haute résistance. L'observation par profilométrie des surfaces suite à une campagne expérimentale a montré que cette technique permettait de retrouver de manière significative le profil d'usure. Hegadekatte [9] a identifié un coefficient d'usure dans le cas d'un contact cylindre-cylindre pour des matériaux céramiques. Le modèle développé a été

Auteur correspondant : n_benghalem@hotmail.com 
implémenté sur le logiciel commercial ABAQUS dont l'une des extensions permet le remaillage automatique et tout ou partie d'une pièce à chaque pas de temps. Le modèle développé a été implémenté sur le logiciel commercial ABAQUS dont l'une des extensions permet le remaillage automatique.

\section{Procédure expérimentale \\ 2.1 Matériaux et éprouvette}

Les éprouvettes sont en acier 25CrMo4, sa composition chimique est donnée dans le tableau let sa microstructure est illustrée par la figure 2. Les éprouvettes sont réalisées par tournage et la partie concernée par le contact se présente sous une forme tronconique, de demi-angle $=$ $45^{\circ}$, avec un méplat de diamètre initial $2 \mathrm{Ri}=2 \mathrm{~mm}$ (Figure1) pour faciliter l'analyse microstructurale et réaliser des essais dans les mêmes conditions initiales. La partie arrière de l'éprouvette est munie d'une gorge pour sa fixation sur le porte échantillon. D'autre part, l'éprouvette est percée d'un trou borgne, de diamètre 2 $\mathrm{mm}$, dont le fond est situé à $2 \mathrm{~mm}$ de la surface du méplat. Ce trou sert de logement à un thermocouple.

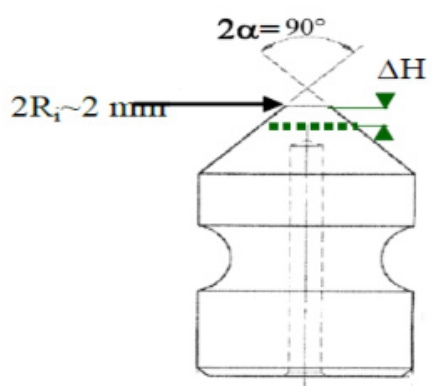

Figure 1. Schéma d'éprouvette utilisée et définition de la hauteur d'usure finale $\Delta \mathrm{H}$. [1]

Tableau 1: Composition chimique (en masse) d'acier 25CrMo4

\begin{tabular}{|l|l|l|l|l|l|l|}
\hline Matériaux & Fe & C & Cr & Si & Mo & Mn \\
\hline 25CrMo4 & 96,7 & $\mathbf{0 , 2 2 -}$ & $\mathbf{1 , 4}$ & $\mathbf{0 , 3}$ & $\mathbf{0 , 1 5}-$ & $\mathbf{1}$ \\
& & $\mathbf{0 , 2 9}$ & & & $\mathbf{0 , 3}$ & \\
\hline
\end{tabular}

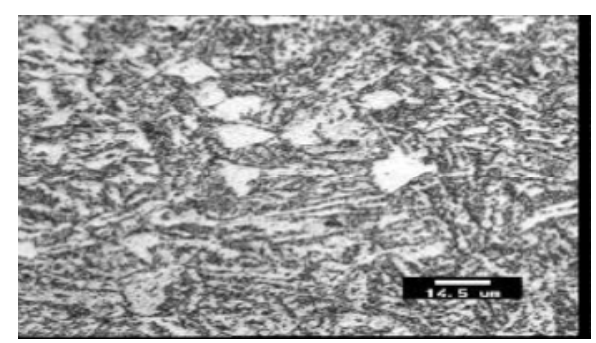

Figure 2. Métallographie de structure initiale d'acier 25CrMo4 (brut) [1]

\subsection{Tribométrie à chaud}

\subsubsection{Description du tribomètre}

Les essais tribologiques à l'ambiante et à chaud ont été effectués sur un tribomètre à chaud (Figure 3 ) du laboratoire de tribologie du centre de mise en forme à Sophia Antipolis (CEMEF). Ce tribomètre est du type pion sur disque. Le disque a un diamètre de $200 \mathrm{~mm}$ et est en carbure de tungstène cémenté au cobalt (grade K20 avec 6\% Co; dureté Hv 1600) ; sa vitesse linéaire périphérique $\mathrm{v}$ peut varier de 0,015 à $0,33 \mathrm{~m} / \mathrm{s}$, sa microstructure est illustrée dans la figure 5. Sa haute dureté et sa faible réactivité chimique dans les conditions des essais garantissent une bonne stabilité des conditions de contact et limite fortement son usure et dégradation. L'éprouvette, située à l'extrémité du bras de levier, est appliquée sur le disque en carbure avec une force normale $\mathrm{P}$, qui peut varier entre 500 et $5000 \mathrm{~N}$. La force est contrôlée par un piston pneumatique et une servovalve. Les forces normales $\mathrm{P}$ et tangentielles $\mathrm{F}$ sont déterminées par des cellules de forces. Un thermocouple est placé à l'intérieur de l'éprouvette et sa soudure chaude est en début d'essai située à $2 \mathrm{~mm}$ de la surface de contact (Figure 4). Il permet de suivre l'évolution de la température macroscopique de la surface de contact au cours de l'expérience. La perte de hauteur h de l'éprouvette (Figure 1) est suivie en continu par un capteur qui mesure le déplacement du porte échantillon dans la direction normale à la surface de contact par rapport au bâti de la machine. $\mathrm{Du}$ fait de divers phénomènes (rattrapage de jeu, dilatation thermique...), nous appelons perte de hauteur apparente, notée h, la valeur finale obtenue par cette mesure qui peut présenter quelques différences avec la perte de hauteur réelle $\mathrm{H}$ de l'éprouvette que nous pouvons mesurer après essai. Nous nommons donc $\mathrm{h}$ la perte de hauteur apparente de l'éprouvette selon le capteur de déplacement. Un four circulaire qui vient se placer autour du disque et de l'éprouvette permet d'effectuer des mesures à chaud jusqu'à $700^{\circ} \mathrm{C}$. Sur ce banc d'essai CEMEF, on mesure donc en continu :

La force normale $\mathrm{P}$, la force tangentielle $\mathrm{F}$, le déplacement progressif de l'éprouvette dû au raccourcissement « apparent» de sa hauteur ou perte de hauteur, h de l'éprouvette, la température de l'éprouvette, L'acquisition des données est assurée toute les 0.45 secondes

\subsubsection{Procédure expérimentale et conditions} d'essais

Le pion appuyé contre le disque par une charge normale $\mathrm{P}$ a une forme cylindrique de longueur $20 \mathrm{~mm}$ et de diamètre égale à $10 \mathrm{~mm}$, contient un trou, qui permet l'introduction d'un thermocouple pour mesurer la température à $2 \mathrm{~mm}$ du contact. Le disque a la forme d'une couronne de diamètre extérieur $200 \mathrm{~mm}$ et de diamètre intérieur à $5 \mathrm{~mm}$ et d'épaisseur égale à $20 \mathrm{~mm}$. 
Les essais ont été effectués dans les conditions suivantes :

- $\quad$ La force normale varie entre 500 et $1000 \mathrm{~N}$ ce qui correspond à des pressions de contact initiales respectives 160 et $220 \mathrm{MPa}$.

- La vitesse de glissement a été maintenue constante pour tous les essais $\mathrm{v}=0,1 \mathrm{~m} / \mathrm{s}$,

- une lubrification continue goutte à goutte.

- La température nominale des essais (température four) varie entre 20 et $200^{\circ} \mathrm{C}$.

- La distance de glissement est fixée à $90 \mathrm{~m}$, ce qui correspond à une durée des essais égale à $15 \mathrm{~min}$ pour $\mathrm{v}=0,1 \mathrm{~m} / \mathrm{s}$.

- La lubrification s'effectue par un essuyage du disque en WC-Co avant le déroulement de l'essai suivie par suivie par une lubrification continue goutte à goutte.

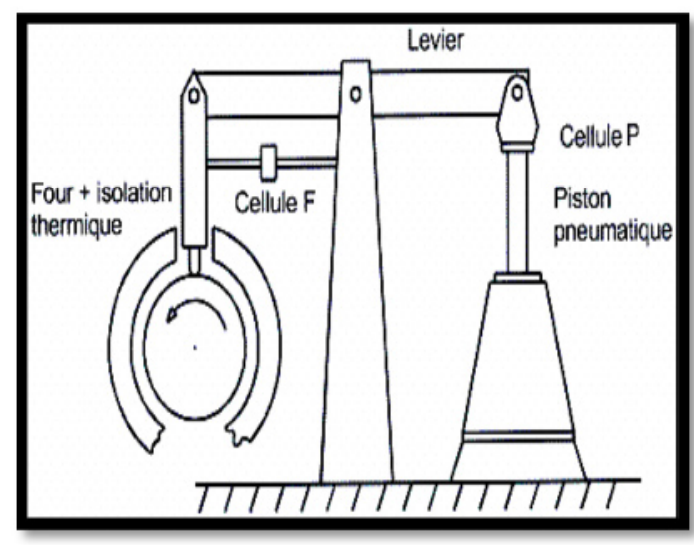

Figure 3. : Schéma du tribomètre à chaud $(\mathrm{P}$ : force normale, $\mathrm{F}$ : force tangentielle) [1]

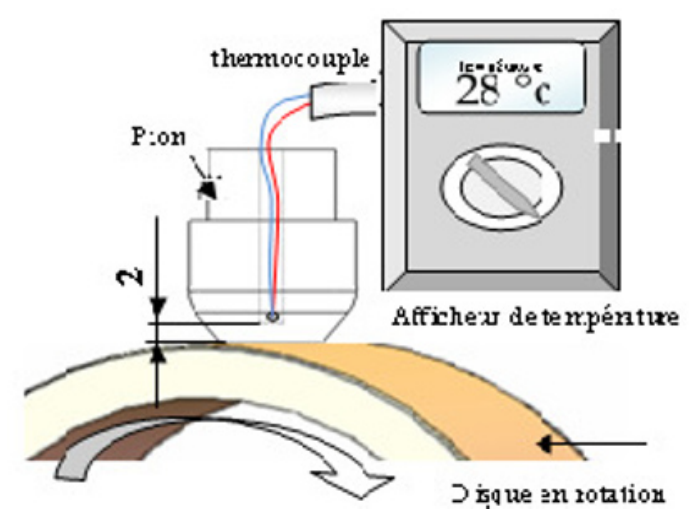

Figure 4 : Mesure de température du couple en contact animé moyennant un thermocouple

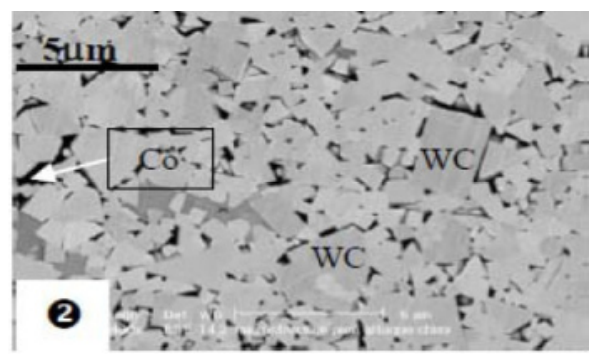

Figure 5 : Microstructure du carbure de tungstène fritté WC-6\%Co. [10]

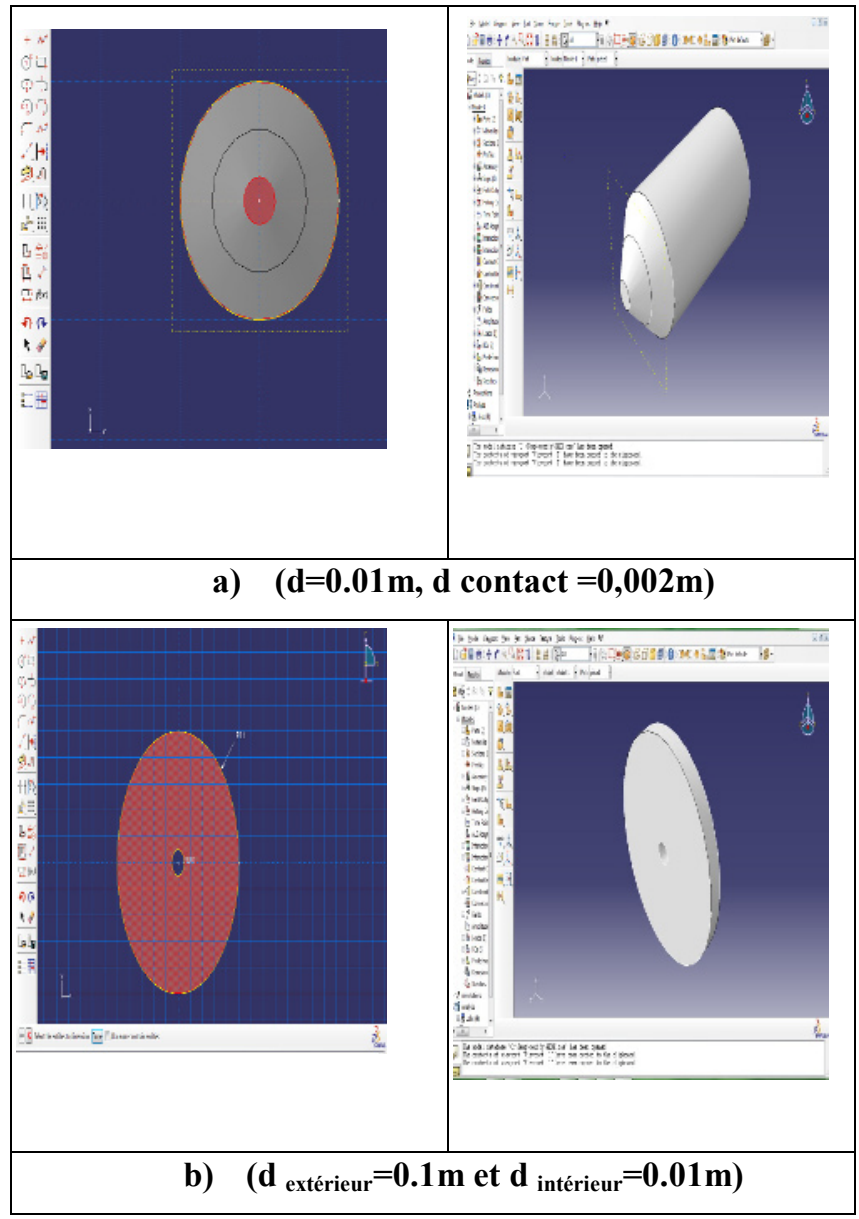

Figure 6 : Géométrie simulée du a) Pion et b) disque

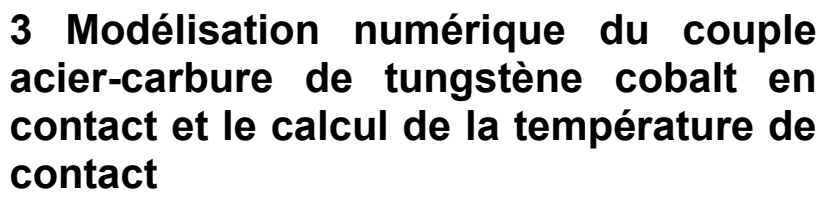




\subsection{Les étapes des entrées des données sur ABAQUS}

La température expérimentale de contact n'est pas facile à déterminer et nécessite la mise en œuvre des moyens d'essais importants. En fait, il est impossible de s'approcher suffisamment de l'interface de contact. Ce qui nous à conduit à utiliser un logiciel de simulation numérique Abacus, qui s'appuie sur un certain nombre de constantes qui caractérisent les propriétés mécaniques et thermiques des matériaux en contact, pour déterminer la distribution du champ de température dans tout le domaine et particulièrement au point de contact. Dans cette partie nous présenterons une modélisation numérique du couple tribologique pion-disque, afin de pouvoir évaluer la température de contact en utilisant le logiciel Abacus. L'objet de ce calcul détermine les températures moyennes de contact à partir des coefficients de frottement du couple frotté et les comparer aux températures expérimentales déterminées à $2 \mathrm{~mm}$ pour le même couple en acier et carbure de tungstènecobalt.

\subsection{Création du profil et du modèle géométrique du pion et du disque}

Dans ABAQUS, pour créer un objet 3D, il est nécessaire de commencer par créer tout d'abord le profil de l'objet. Ensuite ce profil générera l'objet 3D par extrusion. Après la création des modèles géométriques à partir de dimension de données. Une fois les géométries en place, on pout lancer l'étude thermique (voir figure $6 \mathrm{a}$ et b).

\subsubsection{Entrée des propriétés mécaniques et physiques des matériaux utilisés}

Le passage à l'étude thermique nous conduit à faire entrer les caractéristiques des deux matériaux comme le (module élastique, conductivité, chaleur spécifique, dilatation thermique, densité) selon le tableau 2

\subsubsection{Assemblage des pièces (action réciproque entre les pièces)}

Dans cette étape on passe à l'assemblage du pion et du disque. On crée un point de référence sur le disque (dans le centre du disque).

\subsubsection{Définition des pas d'analyse (création du step)}

On sélectionne la région pour laquelle ABAQUS va générer les données. On sélectionne aussi les variables. Et on choisit toutes sortes de paramètres pour générer des données de sortie.

\subsubsection{Création de l'interaction}

Créer l'interaction : c'est définir le contact entre le disque et le pion

\subsubsection{Création de charge et création des conditions aux limites}

Toutes les conditions sont dépendantes du pas d'analyse, c'est-à-dire qu'elles s'activeront à un pas donné

\subsubsection{Maillage}

Le résultat de cette étape est illustré dans la figure $8 \mathrm{c}$

\subsubsection{Etape de calcul}

Cette étape consiste à passer à celle de l'exploitation des résultats et le calcul des données désirées voir figure $8 \mathrm{~d}$

Tableau3 : Propriétés mécaniques et physiques de l'acier et du carbure de tungstène

\begin{tabular}{|c|c|c|}
\hline Caractéristiques & Acier & WC-Co \\
\hline $\begin{array}{l}\text { Module d'élasticité } \\
\text { E }\left(\mathrm{N} / \mathrm{m}^{2}\right)\end{array}$ & $210^{*} 10^{9}$ & $600 * 10^{9}$ \\
\hline $\begin{array}{l}\text { Masse volumique } \\
\rho\left(\mathrm{Kg} / \mathrm{m}^{3}\right)\end{array}$ & 7800 & 14900 \\
\hline $\begin{array}{l}\text { Chaleur spécifique } \\
\mathrm{C}\left(\mathrm{j} / \mathrm{Kg} \cdot \mathrm{k}^{-1}\right)\end{array}$ & 440 & 480 \\
\hline $\begin{array}{l}\text { Conductivité thermique } \\
\mathrm{K}(\mathrm{W} / \mathrm{m} \cdot \mathrm{k})\end{array}$ & 14 & 80 \\
\hline $\operatorname{Re}(\mathrm{MPa})^{*}$ & $450 / 700$ & 1600 \\
\hline $\mathrm{Rm}(\mathrm{MPa})^{*}$ & $700 / 1000$ & 1440 \\
\hline $\mathrm{HV}(2 \mathrm{~N})$ & $220-230$ & 1600 \\
\hline Résistivité ( $\mu \Omega . c m)$ & $10.1 * 10^{3}$ & \\
\hline $\begin{array}{l}\text { Coefficient de dilatation } \\
\text { thermique }\end{array}$ & $1.1 * 10^{-5} \mathrm{~K}^{-1}$ & $\begin{array}{l}5 * 10^{-6} \mathrm{~K}^{-} \\
1\end{array}$ \\
\hline Coefficient de Poisson $v$ & 0.3 & 0.28 \\
\hline Diffusivité $\alpha\left(\mathrm{m}^{2} / \mathrm{s}\right)$ & $1.3 * 10^{-5}$ & \\
\hline $\begin{array}{l}\text { Température de fusion } \\
{ }^{\circ} \mathrm{K}\end{array}$ & 1853 & 3140 \\
\hline
\end{tabular}




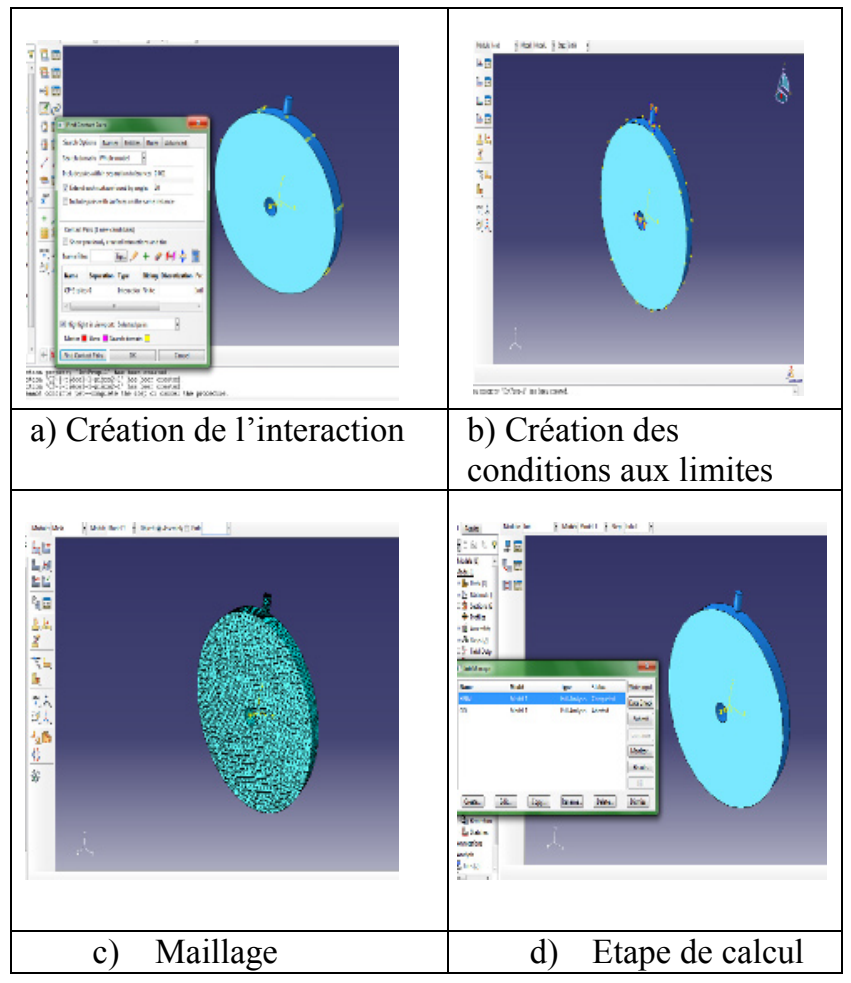

Figure 7 : Illustration en image des résultats des étapes des entrées de données

\section{Résultats}

\subsection{Visualisation des résultats du frottement}

\subsubsection{Résultats obtenus par l'ABAQUS}

Les résultats de simulation de la température de contact en comparaison avec les résultats de la première série d'essai sont indiqués dans le tableau 3 et illustrés par les figures 8et 9

Tableau 3 : Température expérimentale et simulée et leur écart pour $\mathrm{P}=500 \mathrm{~N}$

\begin{tabular}{|l|l|l|l|l|}
\hline Eprouvette & $\mu$ & $\begin{array}{l}\mathrm{T} \text { Exp } \\
{\left[{ }^{\circ} \mathrm{C}\right]}\end{array}$ & $\begin{array}{l}\text { T Sim } \\
{\left[{ }^{\circ} \mathrm{C}\right]}\end{array}$ & $\begin{array}{l}\Delta \mathrm{T} \\
{\left[{ }^{\circ} \mathrm{C}\right]}\end{array}$ \\
\hline A1 & 0,28 & 20 & 34 & 14 \\
\hline A2 & 0,4 & 60 & 80 & 20 \\
\hline A3 & 0,7 & 100 & 131 & 31 \\
\hline A4 & 0,8 & 150 & 184 & 34 \\
\hline
\end{tabular}

\subsubsection{Ecart entre la température expérimentale et simulée}

La figure 8 montre l'intérêt d'identifier numériquement la température de contact. En effet, pour l'essai le moins énergétique, la différence entre la température surfacique calculée $\mathrm{Tc}$ et la température mesurée Te par le thermocouple à $2 \mathrm{~mm}$ sous la surface est égale à $14{ }^{\circ} \mathrm{C}$ en fin d'essai. On remarque nettement d'après la figure 8 que l'allure de la température simulée se trouve en haut de celle de la température expérimentale selon le coefficient de frottement et la force normale appliquée. $\mathrm{La}$ différence entre la température simulée et expérimentale se situe dans l'intervalle $\left[14-34^{\circ} \mathrm{C}\right]$. On note aussi que l'écart augmente lorsque le coefficient de frottement augmente. Cela peut être expliqué par le fait que lorsque le coefficient de frottement augmente l'énergie dissipée au niveau du contact et la déformation plastique augmentent aussi. On rappelle que la température relevée pendant l'essai a été contrôlée par le thermocouple qui est placé à l'intérieur du pion à $2 \mathrm{~mm}$ jusqu'a la fin de l'essai, la température calculée se trouve au niveau de la zone de contact, la déformation s'élabore uniquement sur le pion parce qu'il est moins dur que le disque.

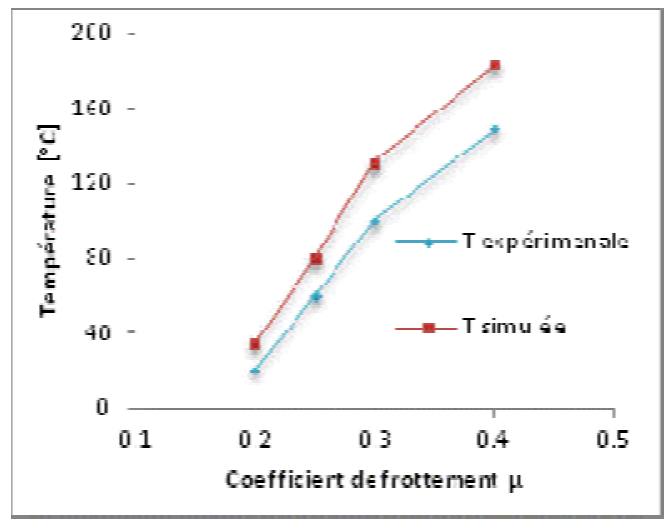

Figure 8: Ecart entre la température simulée et la température expérimentale en fonction du coefficient de frottement, $[\mathrm{v}=0.1 \mathrm{~m} / \mathrm{s}$ et $\mathrm{P}=500 \mathrm{~N}]$

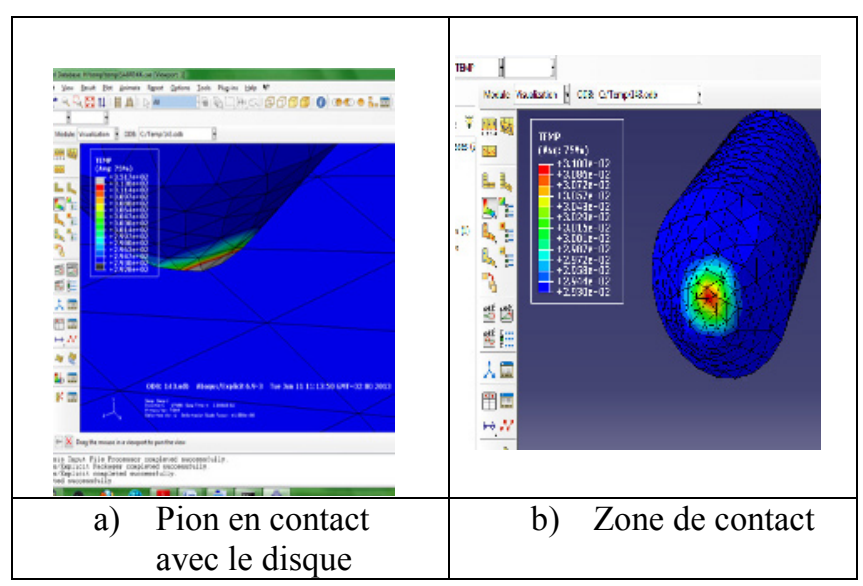

Figure 9 : Effet de la température et forme de la surface de contact du pion après frottement 
Tableau 4 : Les résultats de simulation de la température de contact en fonction du coefficient de frottement sous une charge constante égale à $1000 \mathrm{~N}$.

\begin{tabular}{|c|c|c|c|c|}
\hline Eprouvette & $\boldsymbol{\mu}$ & $\mathbf{T ~ e x p}{ }^{\circ} \mathrm{C}$ & $\mathbf{T ~ s i m}^{\circ} \mathrm{C}$ & $\boldsymbol{\Delta T}$ \\
\hline B1 & 0,12 & 20 & 31 & 11 \\
\hline B2 & 0,14 & 40 & 52 & 12 \\
\hline B3 & 0,18 & 60 & 73 & 13 \\
\hline B4 & 0,27 & 80 & 100 & 20 \\
\hline B5 & 0,29 & 100 & 123 & 23 \\
\hline B6 & 0,29 & 120 & 154 & 34 \\
\hline B7 & 0,67 & 140 & 179 & 39 \\
\hline B8 & 0,65 & 160 & 198 & 38 \\
\hline B9 & 0,6 & 180 & 218 & 38 \\
\hline B10 & 0,58 & 200 & 239 & 39 \\
\hline
\end{tabular}

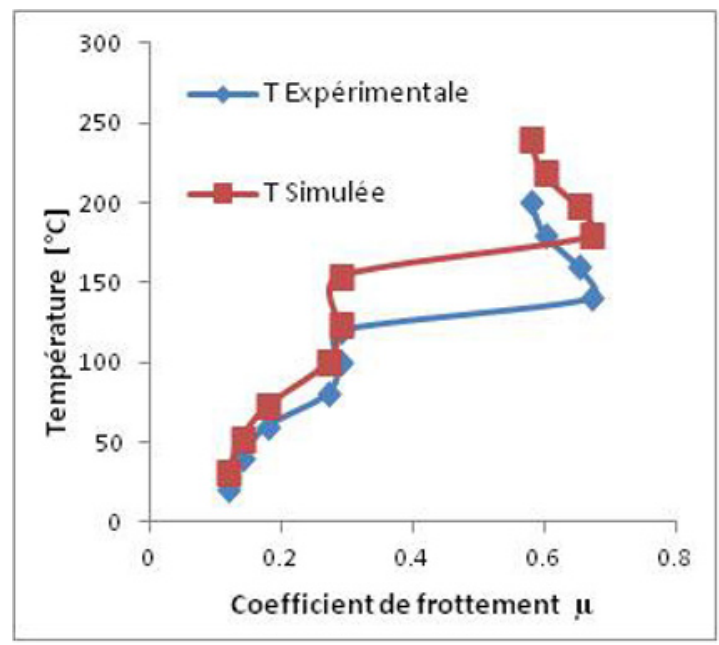

Figure 10: Ecart entre la température expérimentale et la température simulée en fonction du coefficient de frottement avec $[\mathrm{v}=0.1 \mathrm{~m} / \mathrm{s}$ et $\mathrm{P}=1000 \mathrm{~N}]$

La figure 10 montre l'écart entre les deux températures respectivement expérimentale et simulée, on remarque que la courbe de la température simulée est en haut par rapport à celle donnée par l'expérimentation, c'est-à-dire que les valeurs de la température simulée sont plus grandes que celles recueillies par l'expérimental. On observe deux stades distincts, dans le premier stade et pour les températures allant de $\left[20-100^{\circ} \mathrm{C}\right]$ l'écart est petit et se localise dans la plage $\left[11-23^{\circ} \mathrm{C}\right]$, par contre dans le second l'écart se situe dans l'intervalle [34-39
${ }^{\circ} \mathrm{C}$ ], cependant l'écart est plus élevé et l'étendue est plus faible de $1^{\prime}$ 'ordre de $5^{\circ} \mathrm{C}$. On calcule un écart de $28^{\circ} \mathrm{C}$ entre la valeur minimale et maximale de température. Ces écarts restent acceptables étant donné que les valeurs théoriques et pratiques présentent des lieux de prise différents. Le lieu de la température expérimentale est situé à $2 \mathrm{~mm}$ du contact et celui de la température simulée est pris exactement au point de contact.

\section{Conclusion}

Nous avons visé par ce travail à apporter une amélioration significative à la compréhension et à la simulation des phénomènes tribologiques à l'interface pion/disque lors d'une opération de frottement et de recueillement de température de contact.

A travers cette étude, nous avons utilisé pour le travail expérimental un tribomètre pour déterminer le frottement et la température de contact entre pion-disque (acier «25CrMo4» contre carbure de tungstène-cobalt) par contre pour la simulation on a utilisé le logiciel numérique (ABAQUS) qui permet de calculer, de trouver la température au niveau du contact et de simplifier la durée de calcul, consacrée essentiellement à l'analyse des aspects thermiques d'une interface de contact. Ce travail a pour objectif principal de déterminer la température de l'interface de contact d'un pion sur disque en mouvement relatif. L'intérêt de connaître la température de contact d'un corps en frottement réside dans le fait que cette température est susceptible de modifier les propriétés physicochimiques des corps en contact ce qui peut conduire à la dégradation de l'interface.

Les résultats que nous avons obtenus peuvent être résumés comme suit :

- La température de contact augmente proportionnellement avec les forces normales.

- La différence entre la température simulée et la température expérimentale varie entre [11$39^{\circ} \mathrm{C}$ ] selon la température initiale et les autres paramètres (la vitesse, la charge, l'état de surface, l'aire de contact, lubrifiants, la nature des matériaux utilisés et la géométrie).

- La température de contact augmente au niveau de la surface du pion et non pas à la surface du disque, puisque toute l'énergie va être transmise au pion.

- La simulation numérique est une méthode de calcul qui réduit le temps de l'étude des comportements mécaniques et thermiques des matériaux et qui nous informe davantage sur les problèmes inaccessibles en expérimental. 


\section{Références}

[1] N. Khanafi-Benghalem, «Etude des mécanismes d'usure et de frottement de deux aciers X12CrNiMo25-20 et le 25CrMo4 (étude comparative) ", Thèse de doctorat université Ferhat Abbas Sétif- ALGERIE, PP 142 (2007),

[2] D. Meresse, "Approche thermomécanique de la tribologie à grande vitesse Application au freinage", Thèse de doctorat Lille Nord-France, pp151, (2011)

[3] N. Khanafi-Benghalem, K. Loucif, K. Benghalem, H. Boudoukha and R. Louahdi, "Contrôle de la température durant le processus de dégradation d'un acier Z12CNS20 en frottement avec un acier Z200C13», J. Phys. IV France 124, pp189-194,(2005)

[4] A. Anderson. "Hot spotting in automotive friction systems», Wear, Vol. 135, No. 2, pp. 319-337, (1990.),

[5] N. Laraqi, N. Alilat, J. G. de Maria, and A. Baïri. "Temperature and division of heat in a pin-on-disc frictional device-Exact analytical solution» Wear, Vol. 266, No. 7-8, pp. 765-770, (2009)

[6] D. Christian, «Méthode des éléments finis», Université de Cergy Pontoise France, mémoire de fin d'étude en mathématique, PP 94, (2006)

[7] M. Siroux, A. L. Cristol-Bulthe, Y. Desplanques, B. Desmet, and G. Degallaix, «Thermal analysis of periodic sliding contact on a braking tribometer», Applied Thermal Engineering, Vol. 28, No. 17-18, pp. 21942202, (2008)

[8] I. McColl, «Finite element simulation and experimental validation of fretting wear». Wear, Vol. 256, No. 11-12, pp. 1114-1127, (2004),

[9] V. Hegadekatte, S. Kurzenhauser, N. Huber, and O. Kraft, «A predictive modelling scheme for wear in tribometers», Tribology International, Vol. 41, No. 11, pp. 1020-1031,(2008)

[10] D. Bouvard, «Métallurgie des poudres ", Thèse de doctorat, Paris: Lavoisier, PP 377,(2002), 